\title{
Ultrafast Surface Inspection System based on Image Processing with Cellular Neural Networks (CNN)
}

Blug, Andreas; Jetter, Volker; Strohm, Peter; Carl, Daniel; Höfler, Heinrich

Fraunhofer-Institut für Physikalische Messtechnik IPM

Heidenhofstr. 8, D-79110, Freiburg, Germany

\begin{abstract}
An important issue in industrial quality assurance is the inspection of rapidly moving surfaces for small defects such as scratches, dents, grooves, or chatter marks. This paper describes an image processing system based on a novel camera technique - the so called Cellular Neural Networks (CNN) for an industrial application which was not feasible for conventional machine vision systems so far. An example for such an application is the $100 \%$ surface control in the production of aluminum wires where defects with a lateral size of $100 \mu \mathrm{m}$ have to be detected at feeding rates of $10 \mathrm{~m} / \mathrm{s}$.
\end{abstract}

To solve this problem, an image processing system was developed whose key component is a camera based on CNN. CNN can be considered as a technology to integrate a ,Single Instruction Multiple Data' (SIMD) processor architecture in the electronic circuitry of CMOS cameras. The result is a machine vision system based on a camera where every pixel has its own processor and which enables real time processing of up to 5800 images per second with a resolution of $176 \times 144$ pixels. The exposure time which limits the motion blur - is $10 \mu$ s for a dark field illumination. In comparison to line cameras this corresponds to more than one million lines per second if no overlap is taken into account. With a more realistic overlap of $50 \%$ between two succeeding images, the equivalent line rate is still 500,000 lines per second with a resolution of 144 pixels - which is about five times above the acquisition rates achieved with conventional imaging systems.

\section{Introduction}

Wire drawing is a metal working process used to reduce the cross section of a wire by pulling it through a series of drawing dies. The profile of each die is designed to introduce a predefined series of strains which alter the microstructure of the wire material. Therefore, besides a plastic deformation certain material properties such as hardening or surface quality are achieved [1-3]. However, surface deformations can cause unwanted shear forces which waken or even break the wire. As FEM simulations by Fraunhofer IWM show [4], in particular edges extending perpendicular to the wire axis show this effect (Fig. 1). Even rather small edges with a width of $100 \mu \mathrm{m}$ are critical for crack formation. This raises the need for surface inspection systems which are able to detect such defects at typical feeding rates of $10 \mathrm{~m} / \mathrm{s}$.

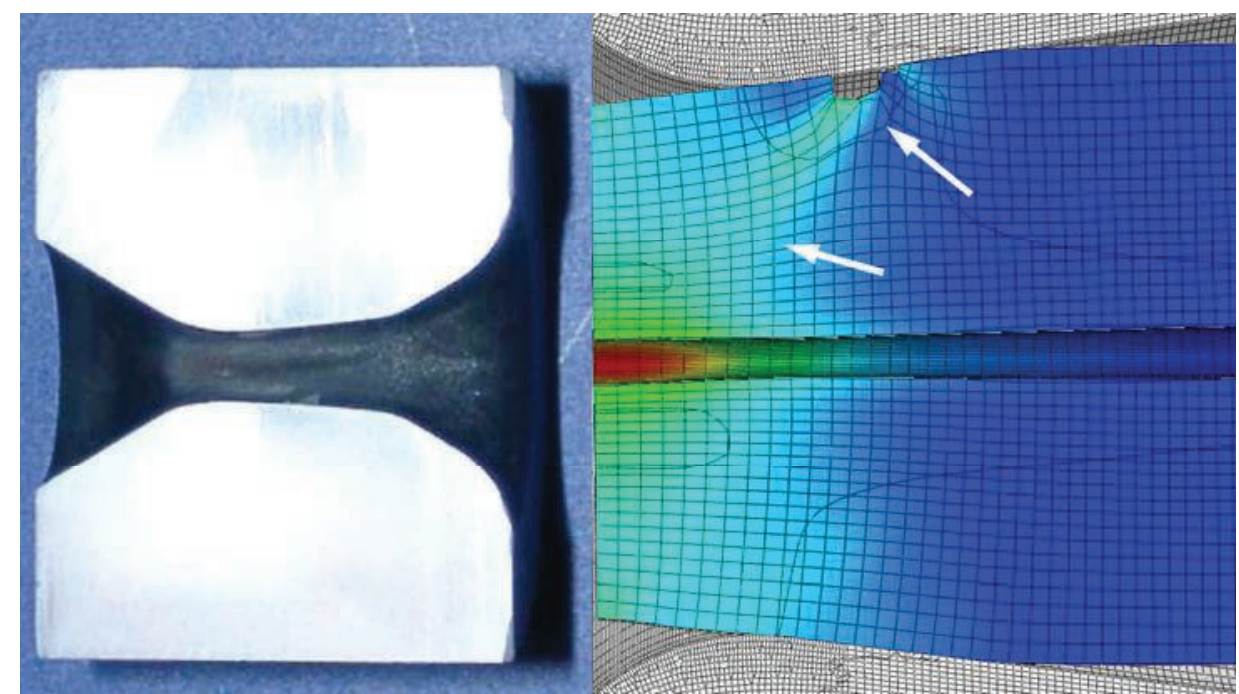

Fig. 1: Left: Cross section of a drawing die with an inner diameter of $3 \mathrm{~mm}$. Right: FEM simulation of shear forces within a wire drawn from right to left [4]. The arrows mark a surface defect extending perpendicular to the wire axis and the resulting shear forces within the bulk material of the wire. 


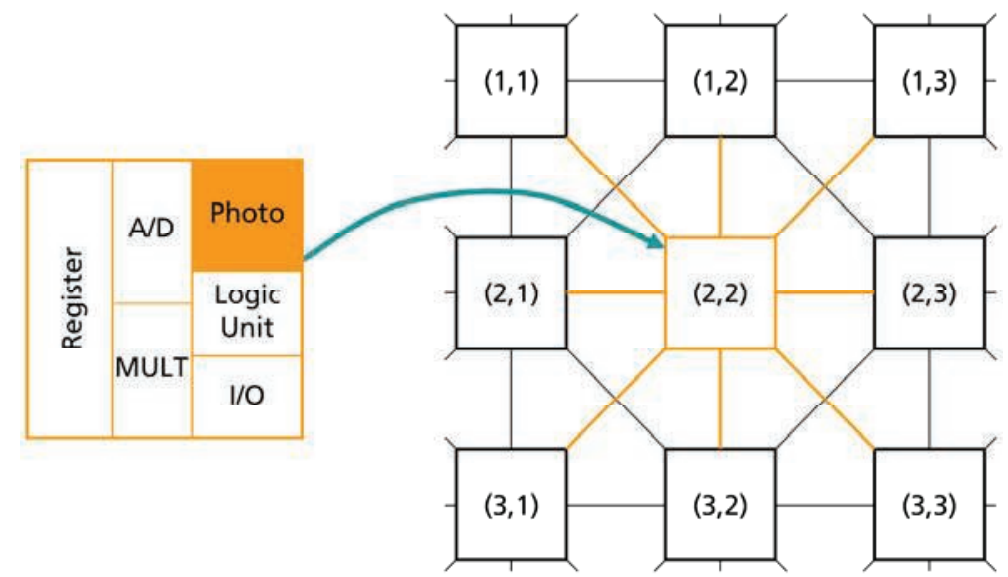

Fig. 2: $\mathrm{CNN}$ enable the integration of processor elements in the electronic circuitry of CMOS cameras.

\section{Cellular Neural Networks (CNN)}

With conventional image processing architectures like line cameras, this system had to acquire and process more than 200,000 lines per second for an optical resolution of $50 \mu \mathrm{m}$, which is not feasible due to two bottlenecks: the camera system and the computing system. Conventional computing systems with the exception of FPGA are usually "Single Instruction Single Data" (SISD) architectures [5]. Here a program consists of a series of instructions which are applied sequentially to single data elements by one or few processor cores. Therefore the execution time lies in the order of $\mathrm{N}$ or $\mathrm{N}^{2}$, where $\mathrm{N}$ is the number of pixels. One alternative are "Single Instruction Multiple Data" (SIMD) computing architectures in which each instruction of a program is applied in parallel to a large number of data. Such SIMD applications are especially efficient for image processing applications which frequently apply the same operation on different pixels of the image. On SIMD systems, these operations can be executed simultaneously in a single step.

Cellular neural networks, which were originally invented by Chua and Yang [6], can be considered as a technology which enables computing elements and storage cells to be integrated into camera pixels. It provides a possible basis for the integration of SIMD architectures into the electronic circuitry of the pixels in CMOS cameras. The CNN universal machine forms the basis [7]. It can be implemented in a small space with the aid of CMOS processes in an array of optical sensors together with analogue and digital data processing elements $[8,9]$. Such systems are spatial arrangements of locally connected, non-linear dynamic units that are usually called cells. In addition to the photo sensor, each of these cells contains storage and computing elements as well as switching elements for the input/output of results (Fig. 2). In this way, cellular neural networks link the intensity values of spatially and temporally adjacent pixels and they are especially efficient for operations exploiting these neighborhoods. Typical examples for neighborhood based operations are morphological and logical operations as we use for the detection of the full penetration hole.

Here, a system called Q-Eye developed by Anafocus [10] was used to execute the algorithms for the detection of the full penetration hole. It consists of $176 \times 144$ cells which are each interconnected to the 8 neighboring cells. The Q-Eye is part of the Eye-RIS camera which contains an additional FPGA based NIOS II processor by Altera to control the operation of the whole vision system and to analyze the information output of the Q-Eye, e.g. performing all the decision-making and actuation tasks.

\section{Measurement Setup}

For the inspection of moving surfaces the optical path of a machine vision system must fulfill a number of criteria: First of all, the light intensity must allow exposure times which are short enough to avoid motion blur. Secondly, focal depth and optical resolution must be sufficient to image the smallest features throughout the measurement volume - this is achieved by large $f$ numbers which contradict short exposure times. In particular, on uneven metallic surfaces specular reflections must be avoided in order to achieve a homogeneous intensity distribution on the "good" object surface and making a high contrast for those features to be observed. Therefore, the lighting is essential for the performance and for the image processing algorithms. The LED based partial dark field illumination system used in the setup was described in detail in [11]. In the meantime, the exposure time was shortened to $10 \mu \mathrm{s}$ in combination with 

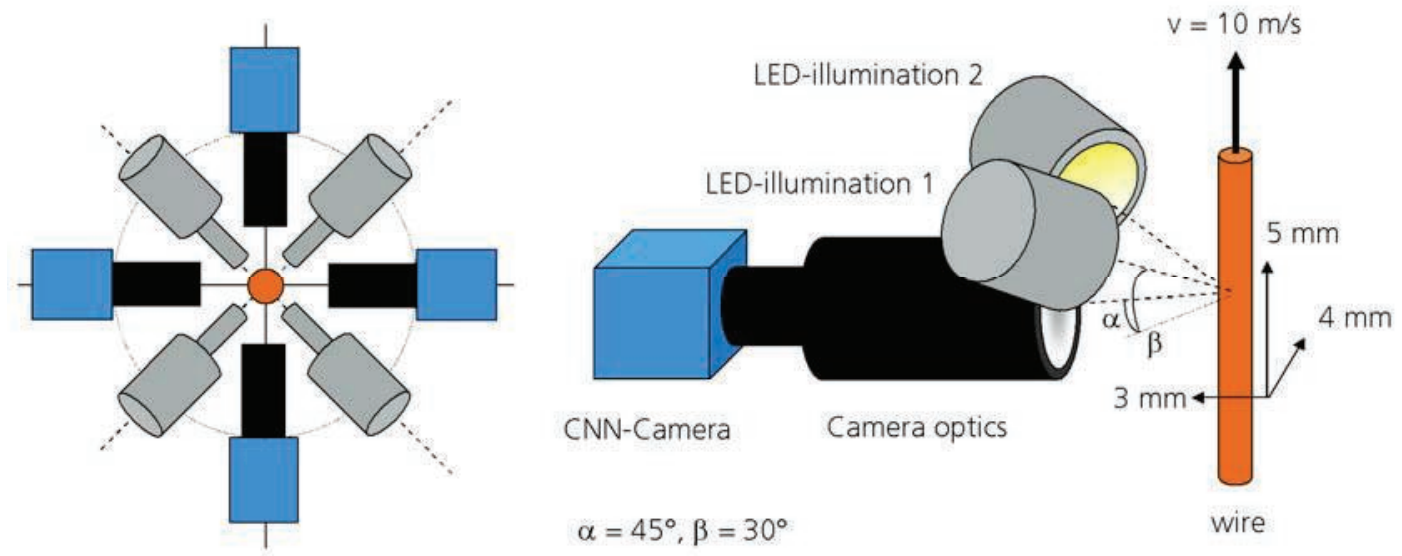

Fig. 3: Measurement setup for a $100 \%$ suface control. Left: Arrangement of four cameras (blue) and illumination systems (grey) around a wire (orange). Right: $90^{\circ}$ section with one camera and two LED lamps for a dark field illumination as used in the test site.

f number 11 for the camera optics. This rather large $f$ number is necessary to maintain the optical resolution beyond $50 \mu \mathrm{m}$ throughout the measurement volume of $5 \times 4 \times 3 \mathrm{~mm}^{3}$.

The test site described in this article is designed to prove the feasibility of a 100 per cent surface surveillance of aluminum wires within the drawing process. As sketched in Fig. 3, the complete setup for production requires a combination of four cameras and four LED illumination units arranged in a 90 degree symmetry. For the proof of feasibility, this setup can be reduced to one camera with two illumination units. Fig. 4 shows this reduced setup in combination with a rotating plate on which the wire samples are mounted. In this way it is possible to simulate feeding rates up to $50 \mathrm{~m} / \mathrm{s}$. As the enlarged inset in fig. 4 shows, the illumination units run in pulsed mode triggered by the camera. This allows image acquisition with sampling rates of up to $10 \mathrm{kHz}$ at exposure times of $10 \mu \mathrm{s}$.

This setup was used to investigate the surface of samples taken from a so called dry aluminum drawing process running without lubricants. The samples were taken from a stage where the wire diameter is reduced from about 3.6 to $3.3 \mathrm{~mm}$ at a feeding rate of $6 \mathrm{~m} / \mathrm{s}$.

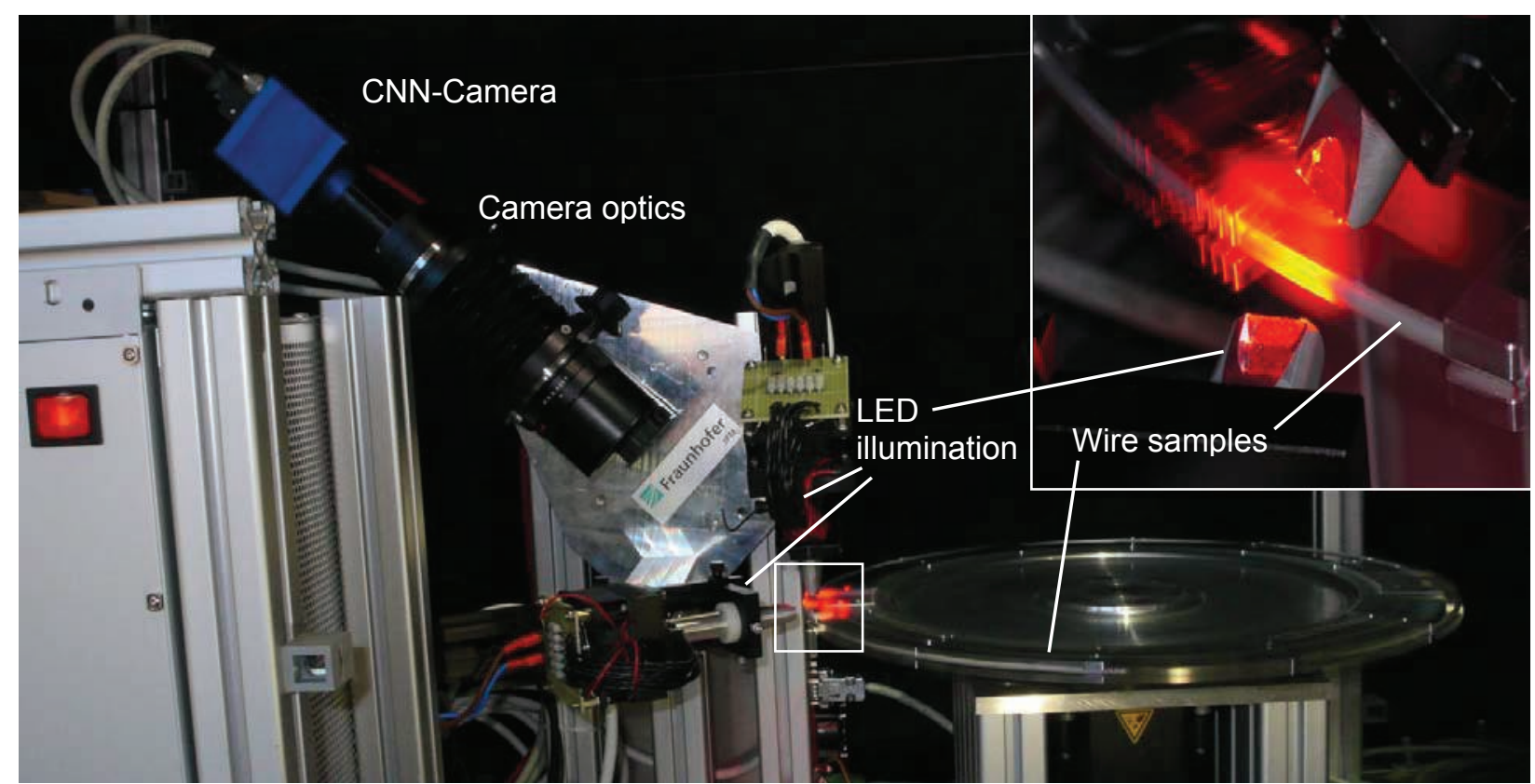

Fig. 4: Photograph of the test site as sketched in Fig. 3. The wire samples are mounted on a rotating disk. As the enlarged inset shows, the illumination units run in pulsed mode with up to $10 \mathrm{kHz}$. 

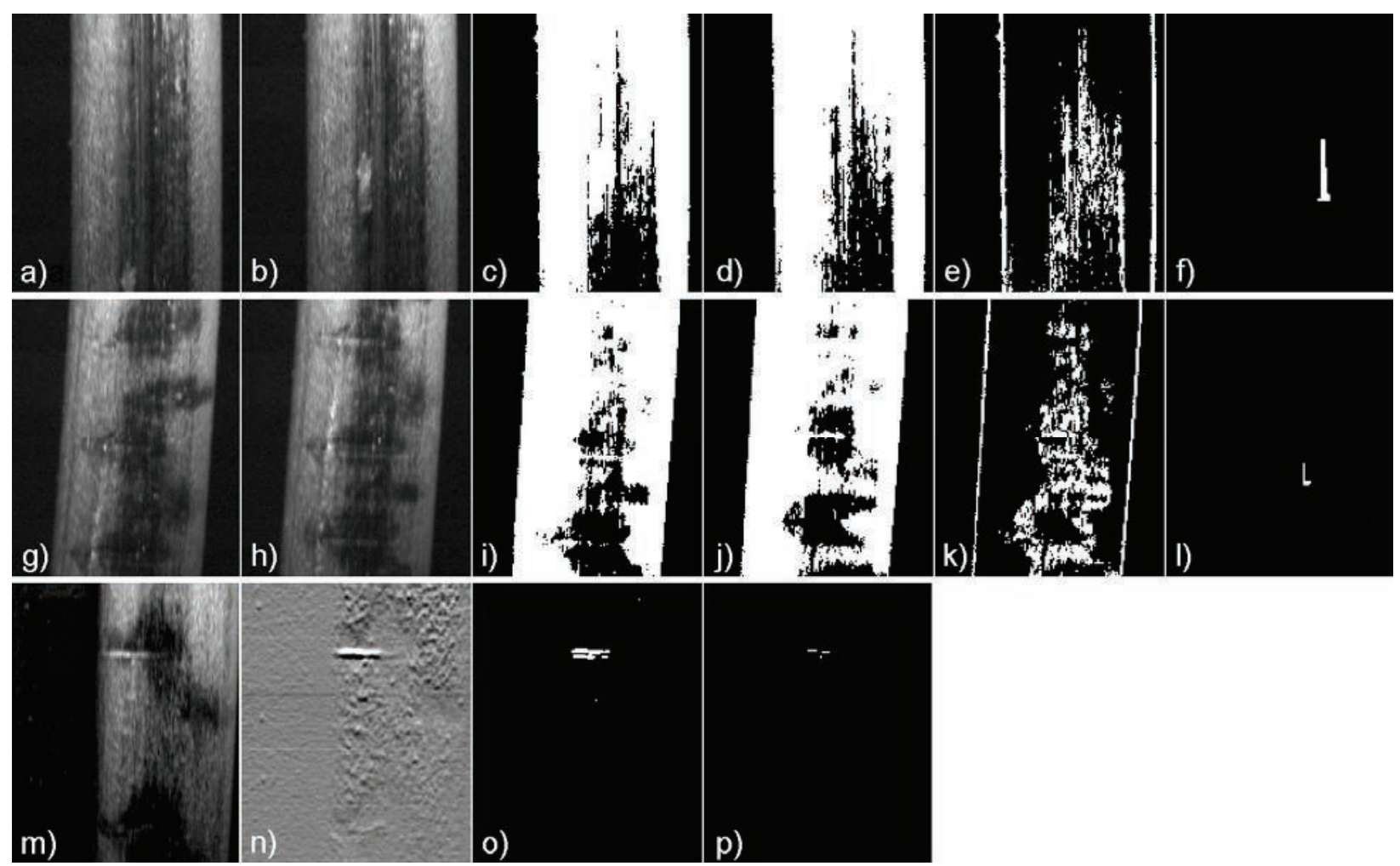

Fig. 5: First row: Two consecutive images a), b) of die marks on the wire with the corresponding threshold images c), d), XOR image e) and final result f) of the first algorithm. Second row: Two consecutive images $\mathrm{g}$ ), h) of chatter marks with the corresponding threshold images i), j), the XOR image $k$ ) and the final image processing result I). Third row: Horizontal dent $m$ ), result image $n$ ) of a horizontal Sobel filter, thresholded Sobel image o), and final result of the second algorithm p).

\section{Defect detection}

In the following, CNN algorithms for the detection of three typical types of defects are described where a control system has to react on in real time. Fig. 5 shows example images together with intermediate and final result images of the corresponding CNN algorithm. The first row shows two consecutive images of marks caused by sharp edges on the surface of a worn drawing die. The overlap between these consecutive images is more than $50 \%$ in order to cover the surface completely. In the beginning, these die marks can be reduced by decreasing the feeding rate. When they increase in size, the process has to be stopped in order to change the dies. In the second row of Fig. 5, chatter marks caused by resonance vibrations within the system are shown. When they occur the control system must react in the same way as on the die marks by reducing the feeding rate. Horizontal dents as the one shown in the bottom are the most critical defects because they may weaken or even break the wire, as described in the introduction. Therefore, the process must be stopped immediately to avoid further damages.

On CNN, algorithms which exploit neighborhoods in space and time are extremely efficient. Therefore, the illumination system was designed in the way that the specular surface of the defects appears darker than the diffuse reflecting "good" wire surface. Both, the die marks and the chatter marks are detected by the same algorithm which is based on the assumption that the intensity of the defect area is less homogeneous than the one of the "good" surface areas. In this way the natural gradient of the dark field illumination is used by applying a global threshold to two consecutive images. Due to their inhomogeneity and to the gradient of the illumination, the lower boundary of the white pixels, where the intensity exceeds the threshold values, will vary in the defect area. These varying areas can be filtered out by a pixel wise XOR connection between the two threshold images. The result is a difference image containing large parts of the defect area and the variation of the border due to the horizontal movement of the wire. To distinguish between the defect and the border, the defect area is closed by five dilation and 17 erosion operations. The effect of the erosion is that the narrow border areas are extinguished. The runtime of this algorithm is about $61 \mu \mathrm{s}$. 
The algorithm for the horizontal dents is simpler. Here, a horizontal Sobel operator is applied in order to enhance the edges of the dents. The result is a line shaped local maximum near to a local minimum. In order to increase the robustness, both, the maximum and the minimum are filtered by a high threshold for the maximum and a low threshold for the local minimum. Since the Sobel filter is a high pass filter, this operation is sensitive to noise. Therefore, both threshold images are combined by a pixel wise OR operation and the noise is filtered out by a single dilation in order to close the contour of the minimum and the maximum and a double erosion to delete the noise. The runtime of this algorithm is about $40 \mu \mathrm{s}$.

These algorithms were tested by a reference series of 454 images acquired with the test site at $6 \mathrm{~m} / \mathrm{s}$. Each of these images was judged visually whether it shows no defect, chatter marks, die marks or horizontal dents. This visual reference was compared to the results of the algorithms above. Among the 268 "good" images five were detected as die or chatter marks by the first algorithm and none was detected as defective by the second algorithm. Since both die marks and chatter marks must also occur in subsequent image, these false positives are easily eliminated in practice. Algorithm one detected all die marks and chatter marks. The reason why the horizontal grooves where detected, too, was, that they all occurred together with chatter marks. Thus, the second algorithm is needed to discriminate these two errors from each other. As the results in table 1 show, this second algorithm did not create false positives. Nevertheless, a comparison between the images $\mathrm{h}$ ) and $\mathrm{m}$ ) in figure 5 shows, the discrimination between the horizontal grooves and the chatter marks is a continuous transition. Thus choosing the parameters of algorithm 2 for a lower sensitivity on chatter marks also reduces the detection rate for horizontal grooves.

\begin{tabular}{|l|c|c|c|c|c|}
\hline Defect & $\begin{array}{c}\text { Number of } \\
\text { reference } \\
\text { images }\end{array}$ & $\begin{array}{c}\text { Detection } \\
\text { rates } \\
\text { algorithm 1 }\end{array}$ & $\begin{array}{c}\text { Algorithm 1, 3 } \\
\text { subsequent } \\
\text { images }\end{array}$ & $\begin{array}{c}\text { Detection } \\
\text { rates } \\
\text { algorithm 2 }\end{array}$ & $\begin{array}{c}\text { Combined } \\
\text { detection } \\
\text { rate [\%] }\end{array}$ \\
\hline $\begin{array}{l}\text { Number of } \\
\text { subsequent images }\end{array}$ & 1 & 3 & 1 & \\
\hline No defect & 268 & 5 & 0 & 0 & $\mathbf{0}$ \\
\hline Chatter marks & 67 & 67 & 67 & 4 & $\mathbf{1 0 0}$ \\
\hline Die marks & 92 & 92 & 92 & 24 & $\mathbf{1 0 0}$ \\
\hline Horizontal grooves & 27 & 27 & 0 & 23 & $\mathbf{8 5}$ \\
\hline $\begin{array}{l}\text { Total number of } \\
\text { images }\end{array}$ & $\mathbf{4 5 4}$ & & & & \\
\hline
\end{tabular}

Table 1: Algorithm results for the reference series acquired with the test site shown in figure 4 .

For a practical industrial application the defects falsely detected on good surfaces are the most critical issue. Compared to such an application, the number of defects is already over weighted in the reference series. Therefore, the $2 \%$ false detections must be reduced. As mentioned above, this can be achieved by taking subsequent images into account. The results listed in table 1 for three subsequent images were obtained by the requirement, that a defect is detected by algorithm 1 in three subsequent images. Both, die marks and chatter marks are large area defects so that their detection is not influenced by this constraint. In contrast to that, the horizontal grooves have a small area so that they appear only in one or two images due to the overlap. Thus the combined detection rate for the die marks and chatter marks is still 100 per cent; the detection rate for the horizontal grooves is $85 \%$. The frame rate for this combined strategy is $5.8 \mathrm{kHz}$.

\section{Conclusion}

This article investigates the applicability of a CNN based camera to an industrial application which is not feasible for conventional image processing systems: The $100 \%$ surface surveillance at wire drawing. There, defects with lateral sizes down to $100 \mu \mathrm{m}$ have to be detected at feeding rates of $10 \mathrm{~m} / \mathrm{s}$. For conventional image processing systems 200,000 lines per second had to be required and evaluated which is not possible with the systems available on the market.

The CNN camera used here is an area camera with $176 \times 144$ pixels. To obtain the same resolution, a maximum field of view of $8 \mathrm{~mm}$ in the direction of the wire is needed. To be on the safe side, it was chosen to be $5 \mathrm{~mm}$. At a feeding rate of $10 \mathrm{~m} / \mathrm{s}$, this means a minimum sampling rate of $2 \mathrm{kHz}$ in order to cover the surface completely. For practical applications, an overlap of $50 \%$ is desirable which corresponds to a sampling rate of $4 \mathrm{kHz}$. For the three types of defects - die marks, chatter marks and horizontal dents - a feeding rate of $5.8 \mathrm{kHz}$ was achieved. This feeding rate was limited by the execution time of the algorithms. The exposure time of the images had to be $10 \mu$ s due to the motion blur. 
Therefore, these requirements are all fulfilled and there is still room for optimization. With faster algorithms as demonstrated for the laser welding application sampling rates of up to $10 \mathrm{kHz}$ appear realistic.

In comparison to a conventional image processing system based on line cameras the sampling rate of $5.8 \mathrm{kHz}$ corresponds to more than one million lines per second if no overlap is taken into account. With a more realistic overlap of $50 \%$ between two succeeding images, the equivalent line rate is still 500,000 lines per second with a resolution of 144 pixels - which is about five times above the acquisition rates achieved with conventional imaging systems. Therefore, these results show that the CNN technology has the potential to open up new fields of application for industrial image processing in which rapid processing of images is required in real time.

\section{Acknowledgement}

This work was financed by the Baden-Württemberg Stiftung gGmbH within the project "FlexFormCNN Flexible Steuerung von Umformprozessen mit Cellularen Neuronalen Netzen".

\section{References}

[1] Hu, L. W.: Analysis of die profiles in wire drawing. Journal of the Franklin Institute, Volume 263, Issue 4, April 1957, Pages 317-329

[2] Lo, Sy-Wie; Lu, Yuung-Hwa: Wire drawing dies with prescribed variations of strain rate. Journal of Materials Processing Technology 123 (2002) 212-218

[3] Segal, V.M.: Severe plastic deformation: simple shear versus pure shear. Mater Sci Eng. A338 (2002), pp. 331-344.

[4] Blug, Bernhard. Fraunhofer Institute for Mechanics of Materials IWM. Wöhlerstraße 11, 79108 Freiburg, Germany. Bernhard.Blug@iwm.fraunhofer.de

[5] Flynn, M.: Some Computer Organizations and Their Effectiveness, IEEE Trans. Comput., Vol. C21 (1972), pp. 948

[6] L. O. Chua, L. Yang, "Cellular Neural Networks: Theory", IEEE TRANSACTIONS ON CIRCUITS AND SYSTEMS, VOL. 35, NO. 10, OCT. 1988

[7] T. Roska and L.O. Chua, "The CNN Universal Machine: an analogic array computer", IEEE Transactions on Circuits and Systems-II Vol.40, pp. 163-173, March, 1993

[8] Rodríguez-Vázquez, A., Liñán-Cembrano, G., Carranza, L., Roca-Moreno, E., Carmona-Galán, R., Jiménez-Garrido, F., Domínguez-Castro, R., Espejo Meana, S.: ACE16k: The Third Generation of Mixed-Signal SIMD-CNN ACE Chips Toward VSoCs. IEEE TRANSACTIONS ON CIRCUITS AND SYSTEMS-I: REGULAR PAPERS, VOL. 51, NO. 5, pp. 851, MAY 2004

[9] P. Dudek, P.J. Hicks, "A general-purpose processor per-pixel analog SIMD vision chip", IEEE Transactions on Circuits and Systems I: Regular Papers, Volume 52, Issue 1, pp. 13 - 20, Jan. 2005.

[10] Company Anafocus, Avd. Isaac Newton s/n, Pabellón de Italia, Ático Parque Tecnológico Isla de la Cartuja, 41092 Sevilla, Spain

[11] Blug, A.; Jetter, V.; Strohm, P.; Carl, D.; Höfler, H.: High power LED lighting for CNN based image processing at frame rates of $10 \mathrm{kHz}$. Proceedings IEEE 12th International Workshop on Cellular Nanoscale Networks and their Applications (CNNA 2010), 3-5 Feb. 2010, Berkeley, CA, USA.

[12] A. Blug, F. Abt, L. Nicolosi, D. Carl, F. Dausinger, H. Höfler, R. Tetzlaff, R. Weber: CLOSED LOOP CONTROL OF LASER WELDING PROCESSES USING CELLULAR NEURAL NETWORKCAMERAS: MEASUREMENT TECHNOLOGY. Paper 1504, Proc. of 28th International Congress on Applications of Lasers \& Electro-Optics (ICALEO 2009), 2009, Orlando, Florida, USA 